\title{
Microplastics and Nanoplastics in Aquatic Environments: Aggregation, Deposition, and Enhanced Contaminant Transport
}

\section{Supporting Information}

\author{
Environmental Science \& Technology
}

Olubukola S. Alimi, Jeffrey Farner Budarz, Laura M. Hernandez, Nathalie Tufenkji* Department of Chemical Engineering, McGill University, Montreal, Quebec, Canada H3A 0C5

* Corresponding Author. Phone: (514) 398-2999; Fax: (514) 398-6678; E-mail: nathalie.tufenkji@mcgill.ca 
Figure S1: Molecular structures of some commonly detected plastics in the environment. (Adapted from Quinn and Crawford, 2016)
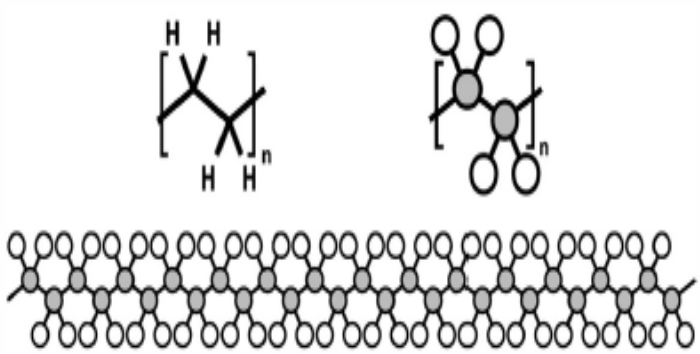

Polyethylene (PE)
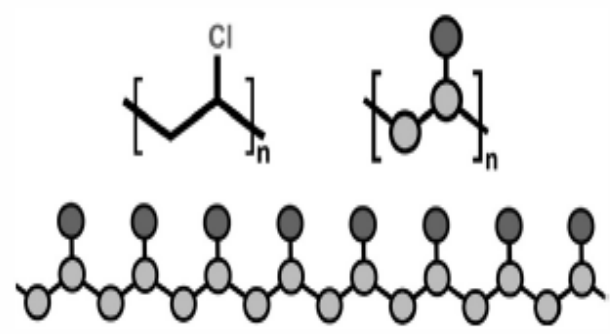

Polyvinyl chloride (PVC)<smiles>c1ccc(C2CC3CCCC(C3)C2)cc1</smiles>

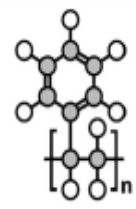

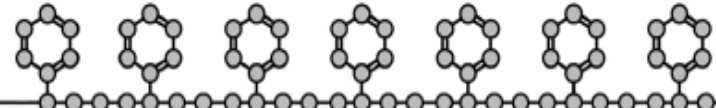<smiles>CC(C)CC(C)CC(C)(C)C</smiles>

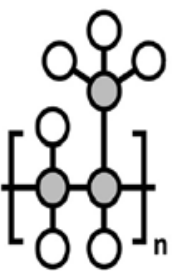

-

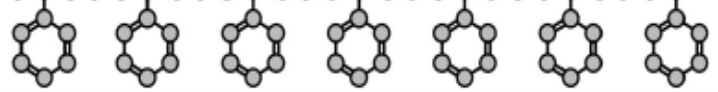
Polystyrene (PS)

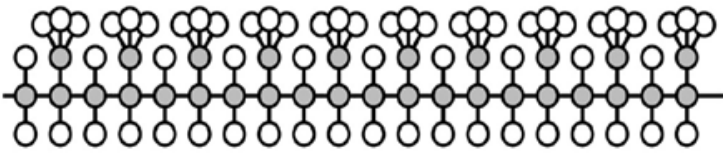

Polypropylene (PP)
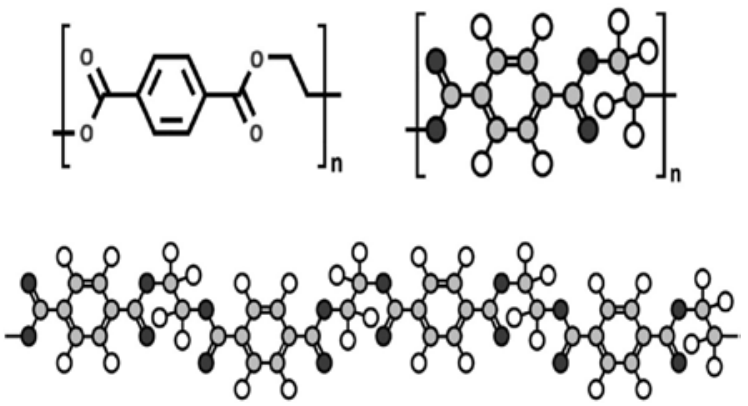

Polyethylene terephthalate (PET) 
Table S1. Data and references for Figure 1

\begin{tabular}{|c|c|c|c|}
\hline Compartment leaving & Compartment entering & $\%$ of Plastic leaving compartment & Reference \\
\hline Manufacture \& Use & Mishandled (Ag \& Land + Lakes \& Rivers) & $25.6-28.0 \%$ & $\begin{array}{l}\text { Calculated from } \\
\text { Jambeck et al., } 2015\end{array}$ \\
\hline Manufacture \& Use & Agriculture \& Land & $0.5-5 \%$ & $\begin{array}{l}\text { Kyrikou and Briassoulis, } 2007 \text {; } \\
\text { Scarascia-Mugnozza et al 2011; } \\
\text { Sintim and Flury, 2017; Maliconico, } \\
2017\end{array}$ \\
\hline Manufacture \& Use & Landfill & $21-42 \%$ & Nizzetto et al., 2016 \\
\hline Manufacture \& Use & WWTP & $0.8-4.6 \%$ & Calculated from Nizzetto et al., 2017 \\
\hline Manufacture \& Use & Oceans & $1.5-4.5 \%$ & Nizzetto et al., 2016 \\
\hline Manufacture \& Use & Recycled & $6-26 \%$ & Barnes et al., 2009; Dris et al., 2015 \\
\hline WWTP & Effluent - Rivers \& Lakes & $* 0.1-5 \%$ (MP only) & $\begin{array}{l}\text { Nizzetto et al., 2017; Horton et al., } \\
\text { 2017; Carr et al., } 2016\end{array}$ \\
\hline WWTP & Solids - Land \& Agriculture & $42-55 \%$ (of sludge) & $\begin{array}{l}\text { Peccia and Westerhoff, 2015; } \\
\text { Samolada and Zabaniotou, } 2013\end{array}$ \\
\hline WWTP & Solids - Landfill & $14-30 \%$ (of sludge) & $\begin{array}{l}\text { Peccia and Westerhoff, 2015; } \\
\text { Samolada and Zabaniotou, } 2013\end{array}$ \\
\hline WWTP & Solids - Incineration & $15-27 \%$ (of sludge) & $\begin{array}{l}\text { Peccia and Westerhoff, } 2015 \\
\text { Samolada and Zabaniotou, } 2013\end{array}$ \\
\hline Agriculture \& Land & Lakes \& Rivers & $(62-84 \%)$ w/ Oceans & Nizzetto et al., 2016 \\
\hline Mishandled & Land \& Agriculture & ${ }^{\dagger}(25.6-28 \%)$ w/ Rivers \& Lakes & Calculated from Jambeck et al., 2015 \\
\hline Agriculture \& Land & Oceans + Lakes \& Rivers & $62-84 \%$ & Nizzetto et al., 2016 \\
\hline Fishing Industry & Oceans & $18-22.3 \%$ & $\begin{array}{l}\text { Andrady, 2011; Ivar do Sul and Costa, } \\
2013\end{array}$ \\
\hline All terrestrial sources & Oceans & $80 \%$ & Andrady, 2011 \\
\hline Lakes \& Rivers & Oceans & $70-80 \%$ (ocean plastics coming from rivers) & Horton et al., 2017 \\
\hline Agriculture \& Land & Oceans & $0-10 \%$ & $\begin{array}{l}\text { Calculated from Andrady, 2011; } \\
\text { Horton et al., } 2017\end{array}$ \\
\hline
\end{tabular}




\begin{tabular}{|c|c|c|c|}
\hline Compartment & Concentration $\left(\# / \mathbf{m}^{2}\right)$ & Concentration ( $\# / \mathbf{L}$ ) & Reference \\
\hline Lakes \& Rivers & $0.020-0.892$ & $0.0024-0.3168$ & Horton et al., 2017 \\
\hline Lakes \& Rivers & & $0.00005-0.032$ & Horton et al., 2017 \\
\hline Lakes \& Rivers & & $4.1373(+/-2.4615)$ & Zhao et al., 2014 \\
\hline Lakes \& Rivers & 0.020 & & EerkesMedrano et al., 2015 \\
\hline Lakes \& Rivers & & 0.000028 & EerkesMedrano et al., 2015 \\
\hline Lakes \& Rivers & $0.01-20$ & & Dris et al., 2016 \\
\hline Lakes \& Rivers & 0.043 & & EerkesMedrano et al., 2015 \\
\hline Lakes \& Rivers & & $0.0003168(+/-0.00046646)$ & Lechner et al., 2014 \\
\hline Lakes \& Rivers & & $0.00194(+/-0.00081)$ & McCormick et al., 2014 \\
\hline Lakes \& Rivers & & $0.01793(+/-0.01105)$ & McCormick et al., 2014 \\
\hline Lakes \& Rivers & 8.465 & & Zhang et al., 2015 \\
\hline Lakes \& Rivers & 3.807 & & Zhang et al., 2015 \\
\hline WWTP Influent & & $260-320$ & Dris et al., 2015 \\
\hline WWTP Effluent & & $14-50$ & Dris et al., 2015 \\
\hline WWTP Influent & & $636.7(+/-38.8)$ & Talvitie et al., 2017 \\
\hline WWTP Effluent & & $3.2(+/-0.7)$ & Talvitie et al., 2017 \\
\hline Sediments & $\sim 0.21-\sim 77,000$ & $0.185-80$ & HidalgoRuz et al., 2012 \\
\hline Sediments & $13,759(+/-16,685)$ & & EerkesMedrano et al., 2015 \\
\hline Sediments & $1108(+/-983)$ & & Imhof et al., 2013 \\
\hline Sediments & $108(+/-55)$ & & Imhof et al., 2013 \\
\hline Sediments & $\sim 0.05-\sim 200$ & & Dris et al., 2016 \\
\hline Sediments & $75-1,300(13,759)$ & & Horton et al., 2017 \\
\hline Agriculture \& Land & & $16-20(+/-6)$ & Browne et al., 2011 \\
\hline Agriculture \& Land & & $4-8(+/-4)$ & Browne et al., 2011 \\
\hline Oceans & & $0.150-2.400$ & Cole et al., 2011 \\
\hline Oceans & $\sim 0.00005-\sim 5$ & $0.000022-8.654$ & HidalgoRuz et al., 2012 \\
\hline Oceans & 0.0269 & & Zhang et al., 2015 \\
\hline Oceans & $10^{\wedge}-6-0.000250$ & & Hinojosa and Thiel, 2009 \\
\hline Oceans & & $0.000167(+/-0.000138)$ & Zhao et al., 2014 \\
\hline
\end{tabular}


Table S2. Summary of sorption studies of contaminants to plastic particles

\begin{tabular}{|c|c|c|c|}
\hline Plastic type and size & Contaminant & Key findings & References \\
\hline PP virgin pellets & $\begin{array}{l}\text { PCBs, DDE, and nonylphenols (NP) } \\
\text { seawater }\end{array}$ & - Contaminants sorb about 1 million times more contaminants than immediate seawater & Mato et al., 2001 \\
\hline $\begin{array}{l}\text { HDPE, PVC } \\
(\mathrm{d}=140 \mu \mathrm{m}, \mathrm{PVC})\end{array}$ & $\begin{array}{l}\text { alkylbenzene; toluene and } \\
\text { o-xylene }\end{array}$ & - $\mathrm{PVC}>\mathrm{HDPE}>$ biopolymers & Wu et al., 2001 \\
\hline LDPE and HDPE & PAH & •HDPE > LDPE (four times more; as a result of the higher surface area of the former) & Muller et al., 2001 \\
\hline PP, PE & PCBs (sea water) & $\cdot P E>P P$ & Mato et al., 2002 \\
\hline Plastic pellets & PCBs & $\begin{array}{l}\text {-Weathered pellets sorbed more PCBs } \\
\text { - PE }>\text { PS } \\
\text {-Sorbed chemicals unevenly distributed among pellets }\end{array}$ & Endo et al., 2005 \\
\hline $\begin{array}{ll}\text { PE } & \left(\mathrm{t}=2.286 \times 10^{-3} \mathrm{~cm}\right) \\
\text { PVC } & \left(\mathrm{t}=1.78 \times 10^{-3} \mathrm{~cm}\right) \\
\text { PS } & \left(\mathrm{t}=3.05 \times 10^{-3} \mathrm{~cm}\right)\end{array}$ & PCBs & $\begin{array}{l}\text { - Average sorption: } \mathrm{PE}>\mathrm{PS}>\mathrm{PVC} \\
\text { - PE showed the highest uptake of } \mathrm{PCBs} \text { and partition coefficients }\end{array}$ & Pascall et al., 2005 \\
\hline $\begin{array}{l}\text { Plastic particles } \\
(\mathrm{d}=200-250 \mu \mathrm{m})\end{array}$ & phenanthrene & $\begin{array}{l}\cdot \text { Desorption rate: sediments }>\text { plastics } \\
\cdot \mathrm{PE} \gg \mathrm{PP}>\mathrm{PVC}>\text { natural sediments }\end{array}$ & Teuten et al.,2007 \\
\hline $\begin{array}{l}\text { PE } \quad(\mathrm{d}=2-3 \mathrm{~mm}) \\
\text { PP } \quad(\mathrm{d}=2-3 \mathrm{~mm}) \\
\text { POM } \quad(\mathrm{d}<2 \mathrm{~mm}) \\
\text { Plastic eroded pellets (PEP) }\end{array}$ & $\begin{array}{l}\text { phenanthrene } \\
\text { synthetic fresh water }\end{array}$ & $\begin{array}{l}\text { - Equilibrium distribution coefficient: } \mathrm{PEP}>\mathrm{PE}>\mathrm{POM}>\mathrm{PP} \\
\cdot \text { Phenanthrene partitions to plastic debris several magnitudes over seawater }\end{array}$ & $\begin{array}{l}\text { Karapanagioti and } \\
\text { Klontza, } 2008\end{array}$ \\
\hline $\begin{array}{l}\text { HDPE,MDPE, LDPE and } \\
\text { PVC } \\
(0.25 \mathrm{~mm} \text { screen })\end{array}$ & $\begin{array}{l}\text { toluene } \\
\text { (landfill) }\end{array}$ & $\begin{array}{l}\text {-Affinity for contaminants in landfills: Plastic }>\text { lignocellulosic materials } \\
\text { - Fast desorption from rubbery plastics compared to glassy plastics }\end{array}$ & Saquing et al., 2010 \\
\hline $\begin{array}{l}\text { PET, HDPE, LDPE, PVC, } \\
\text { and PP }\end{array}$ & PAHs and PCBs & $\begin{array}{l}\text { - Long term field investigation as opposed to laboratory studies where temperature is controlled } \\
\text {-Sorption of both contaminants consistent: HDPE }>\text { LDPE }>\text { PP }>\text { PET }>\text { PVC }\end{array}$ & $\begin{array}{l}\text { Rochman et al., } \\
\text { 2013(a) }\end{array}$ \\
\hline$P E$ beached and virgin & $\begin{array}{l}\mathrm{Cr}, \mathrm{Co}, \mathrm{Ni}, \mathrm{Cu}, \mathrm{Zn}, \mathrm{Cd} \text { and } \mathrm{Pb} \\
\text { (filtered seawater) }\end{array}$ & $\cdot$ Beached $\mathrm{PE}>$ virgin $\mathrm{PE}$ & Holmes et al., 2012 \\
\hline $\begin{array}{l}\operatorname{HDPE}(\mathrm{w} \times 1 \times \mathrm{h}=4.2 \times 4.7 \times 2.8) \\
\operatorname{LDPE}(\mathrm{w} \times 1 \times \mathrm{h}=4 \times 4.4 \times 2) \\
\mathrm{mm}\end{array}$ & PAHs & LDPE $>$ HDPE & Fries and Zarfl, 2012 \\
\hline $\begin{array}{l}\text { PP,PS,PET,PVC, HDPE, } \\
\text { LDPE } \\
(\mathrm{l}=3 \mathrm{~mm}, \mathrm{~d}=2 \mathrm{~mm})\end{array}$ & $\begin{array}{l}\text { PAHs } \\
\text { (marine water) }\end{array}$ & $\begin{array}{l}\cdot \mathrm{PS} \approx \mathrm{HDPE} \approx \mathrm{LDPE}>\mathrm{PP}>\mathrm{PET} \text { and } \mathrm{PVC} \\
\cdot \mathrm{PS} \text { appears to be a source and sink for PAHs }\end{array}$ & $\begin{array}{l}\text { Rochman et al., } \\
\text { 2013(b) }\end{array}$ \\
\hline $\begin{array}{l}\text { PVC and PE } \\
(200-250 \mu \mathrm{m})\end{array}$ & phenanthrene (Phe) and 4,4'-DDT & $\begin{array}{l}\text {-Contaminant transport rely more on concentration than on salinity } \\
\text {-Phe-PE } \gg \text { DDT-PVC }=\text { DDT-PE } \gg \text { Phe-PVC } \\
\text {-Phe: } \text { PE }>\text { PVC }, \text { DDT: PVC }>\text { PE }\end{array}$ & Bakir et al., 2014 \\
\hline $\begin{array}{l}\text { HDPE, PVC, LDPE and PP } \\
(\mathrm{d}=3 \mathrm{~mm}) \\
\text { PET }(1 \times \mathrm{d}=2 \times 3 \mathrm{~mm})\end{array}$ & $\begin{array}{l}\mathrm{Al}, \mathrm{Cr}, \mathrm{Mn}, \mathrm{Fe}, \mathrm{Co}, \mathrm{Ni}, \mathrm{Zn}, \mathrm{Cd} \text { and } \\
\mathrm{Pb} \\
\text { (sea water) }\end{array}$ & HDPE $<$ LDPE, PVC, PET, PP and PS. & Rochman et al., 2014 \\
\hline $\begin{array}{l}\text { PS, PE and PP } \\
(\mathrm{d}<250 \mu \mathrm{m})\end{array}$ & $\begin{array}{l}\text { PAHs; } \\
\text { HCHs } \\
\text { CBs } \\
\text { (Sea water) }\end{array}$ & $\begin{array}{l}\cdot \text { PS show greater affinity for the three contaminants studied except the few most hydrophobic PAHs; } \\
\cdot \text { HCHs: PP }>\text { PE } \\
\cdot \text { CBs: PE }>\text { PP } \\
\cdot \text { PAHS: PS }>\text { PE }>\text { PP }\end{array}$ & Lee et al., 2014 \\
\hline
\end{tabular}




\begin{tabular}{|c|c|c|c|}
\hline $\begin{array}{ll}\text { PE } & (\mathrm{d}=10-180 \mu \mathrm{m}) \\
\text { PS } & (\mathrm{d}=70 \mathrm{~nm})\end{array}$ & $\mathrm{PCBs}$ & $\begin{array}{l}\cdot \text { Salinity increased sorption for both plastic types } \\
\cdot P S>P E\end{array}$ & $\begin{array}{l}\text { Velzeboer et al., } \\
2014\end{array}$ \\
\hline $\begin{array}{l}\text { PE microbeads } \\
(\mathrm{d}=164-327 \mu \mathrm{m})\end{array}$ & $\begin{array}{l}3 \text { H-phenanthrene } \\
14 \text { C-DDT } \\
\text { (sea water) }\end{array}$ & $\begin{array}{l}\text {-DDT preferentially sorbed than phenanthrene in binary mixture } \\
\text {-Rough particles adsorb higher contaminants than smooth ones. }\end{array}$ & Napper et al, 2015 \\
\hline $\begin{array}{l}\text { PE, PS and PVC } \\
(\mathrm{d}=150,230,250 \mu \mathrm{m})\end{array}$ & $\begin{array}{l}\text { Perfluorochemicals: PFOS and } \\
\text { FOSA }\end{array}$ & $\begin{array}{l}-\mathrm{K}_{\mathrm{d} \text { FOSA }}>\mathrm{K}_{\mathrm{d} \text { pFos }} \\
\cdot \mathrm{pH} \text { and salinity: FOSA unaffected while low values favour PFOS sorption } \\
\cdot \cdot \mathrm{FOSA} \text { : PE }>\text { PVC }>\text { PS }\end{array}$ & Wang et al., 2015 \\
\hline $\operatorname{PE}(\mathrm{d}=3.8 \mathrm{~mm})$ & PAHs & - Sorption capacity: $21^{\circ} \mathrm{C}>10^{\circ} \mathrm{C}$ & $\begin{array}{l}\text { Crawford and Quinn, } \\
2017\end{array}$ \\
\hline PS $(d=70 \mathrm{~nm})$ & $\begin{array}{l}\text { PAHs } \\
\text { (fresh water) }\end{array}$ & - Sorption unaffected by aggregate size & Liu et al., 2016 \\
\hline $\begin{array}{l}\text { PE }(d=152.53 \pm 57.92 \mu \mathrm{m}) \\
\text { PA }(d=109.44 \pm 44.53 \mu \mathrm{m}) \\
\text { PS }(d=168.55 \pm 57.50 \mu \mathrm{m}) \\
\text { PVC }(d=57.64 \pm 26.50 \mu \mathrm{m})\end{array}$ & $\begin{array}{l}\mathrm{n} \text {-Hexane, cyclohexane, benzene, } \\
\text { toluene, chlorobenzene, } \\
\text { ethylbenzoate, and naphthalene }\end{array}$ & -Sorption capacity: $\mathrm{PS}>\mathrm{PVC}>\mathrm{PE}>\mathrm{PA}$ & $\begin{array}{l}\text { Huffer and Hofman, } \\
2016\end{array}$ \\
\hline $\begin{array}{l}\text { PE debris } \\
(\mathrm{d}=250-280 \mu \mathrm{m})\end{array}$ & $\begin{array}{l}\text { PPCPs } \\
\text { Dissolved organic matter (humic } \\
\text { acid): } 0-20 \mathrm{mg} / \mathrm{L}\end{array}$ & $\begin{array}{l}\text { - PPCPs sorbed to plastics according to their hydrophobicity } \\
\text {-Increase in humic acid decreased affinity for only three contaminant. } \\
\text {-Increase in salinity affected sorption of one contaminants and not significant for the rest }\end{array}$ & Wu et al., 2016 \\
\hline $\begin{array}{l}\text { PP } \\
(\mathrm{d}=0.425-0.85 \mathrm{~mm})\end{array}$ & $\begin{array}{l}\text { PCB: } 3,3^{\prime}, 4,4^{\prime} \text {-tetrachlorobiphenyl } \\
\text { (simulated seawater, } 3.5 \% \mathrm{NaCl} \\
\text { solution) } \\
\mathrm{C}_{\mathrm{PCB}}=1 \mathrm{mg} / \mathrm{L}\end{array}$ & $\begin{array}{l}\text {-Sorption capacity increases as particle size and temperature decreases } \\
\text {-Sorption capacity: simulated seawater }>\text { ultrapure water }>\mathrm{n} \text { hexane }\end{array}$ & Zhan et al., 2016 \\
\hline $\begin{array}{l}\text { PVC }(\mathrm{d}=1.6 \times 0.8 \mathrm{~mm}) \\
\text { PS }(\mathrm{d}=0.7-0.9 \mathrm{~mm})\end{array}$ & $\begin{array}{l}\mathrm{Cu} \text { and } \mathrm{Zn} \\
\text { (seawater) }\end{array}$ & $\begin{array}{l}\text { - Maximum concentration of } \mathrm{Cu} \text { and } \mathrm{Zn} \text { greater in PVC than PS } \\
\text { - } \mathrm{Cu} \text { sorbed faster than } \mathrm{Zn} \text { on PVC }\end{array}$ & $\begin{array}{l}\text { Brennecke et al., } \\
2016\end{array}$ \\
\hline $\begin{array}{ll}\text { PE } & (\mathrm{d}=50 \mathrm{~nm}) \\
\mathrm{PS} & (\mathrm{d}=20-40 \mu \mathrm{m})\end{array}$ & lubricating oil & $\begin{array}{l}\text {-Sorption: } \mathrm{PE}>\mathrm{PS} \\
\text {-Sorption capacity: independent of } \mathrm{pH} \text { but increases with salinity and temperature }\end{array}$ & Hu et al., 2017 \\
\hline $\begin{array}{l}\text { PE }(\mathrm{d}=100-150 \mu \mathrm{m}) \\
\text { PS } \\
\text { PVC }\end{array}$ & phenanthrene & -Sorption: PE $>$ PS $>$ PVC $>$ natural sediment & $\begin{array}{l}\text { Wang and Wang, } \\
2017\end{array}$ \\
\hline
\end{tabular}

$\mathrm{d}=$ diameter, $\mathrm{l}=$ length, $\mathrm{t}=$ thickness, $\mathrm{w}=$ width

$\mathrm{PE}$ = polyethylene; $\mathrm{HDPE}=$ high-density polyethylene; $\mathrm{LDPE}$ = low-density polyethylene; $\mathrm{POM}$ = polyoxymethylene; $\mathrm{PP}$ = polypropylene; $\mathrm{PS}$ = polystyrene; $\mathrm{PVC}=$ polyvinyl chloride; $\mathrm{PA}$ = polyamide; $\mathrm{PET}=$ polyethylene terephthalate. $\mathrm{FOSA}=$ Perfluorooctanesulfonamide, $\mathrm{PFOS}$ perfluorooctanesulfonate, $\mathrm{PAH}=$ polycyclic aromatic hydrocarbon, $\mathrm{PPCPs}=$ pharmaceuticals and personal care products, $\mathrm{PCB}=$ polychlorinated biphenyls, $\mathrm{HCH}$ = hexachlorocyclohexane, DDE = dichlorodiphenyldichloroethylene, DDT = dichloro diphenyl trichloroethane 


\section{References}

1. Jambeck, J. R.; Geyer, R.; Wilcox, C.; Siegler, T. R.; Perryman, M.; Andrady, A.; Narayan, R.; Law, K. L., Marine pollution. Plastic waste inputs from land into the ocean. Science (New York, N.Y.) 2015, 347, (6223), 768-71.

2. Kyrikou, I.; Briassoulis, D., Biodegradation of agricultural plastic films: A critical review. J. Polym. Environ. 2007, 15, (2), 125-150.

3. Scarascia, G.; Sica, C.; Russo, G., Plastic materials in European agriculture: Actual use and perspectives. J. of Ag. Eng. - Riv. di Ing. Agr 2011, 42.

4. $\quad$ Sintim, H. Y.; Flury, M., Is biodegradable plastic mulch the solution to agriculture's plastic problem? Environ. Sci. Technol. 2017, 51, (3), 1068-1069.

5. Malinconico, M., Soil degradable bioplastics for a sustainable modern agriculture. 2017.

6. $\quad$ Nizzetto, L.; Bussi, G.; Futter, M. N.; Butterfield, D.; Whitehead, P. G., A theoretical assessment of microplastic transport in river catchments and their retention by soils and river sediments. Environ. Sci. Process Impacts 2016, 18, (8), 1050-9.

7. $\quad$ Nizzetto, L.; Futter, M.; Langaas, S., Are agricultural soils dumps for microplastics of urban origin? Environ. Sci. Technol. 2016, 50, (20), 10777-10779.

8. $\quad$ Barnes, D. K.; Galgani, F.; Thompson, R. C.; Barlaz, M., Accumulation and fragmentation of plastic debris in global environments. Philos. Trans. R. Soc. London, Ser. B 2009, 364, (1526), 1985-98.

9. $\quad$ Dris, R.; Gasperi, J.; Rocher, V.; Saad, M.; Renault, N.; Tassin, B., Microplastic contamination in an urban area: A case study in Greater Paris. Environ. Chem. 2015, 12, (5), 592.

10. Carr, S. A.; Liu, J.; Tesoro, A. G., Transport and fate of microplastic particles in wastewater treatment plants. Water Res. 2016, 91, 174-82.

11. Horton, A. A.; Walton, A.; Spurgeon, D. J.; Lahive, E.; Svendsen, C., Microplastics in freshwater and terrestrial environments: Evaluating the current understanding to identify the knowledge gaps and future research priorities. Sci. Total Environ. 2017, 586, 127-141.

12. Samolada, M. C.; Zabaniotou, A. A., Comparative assessment of municipal sewage sludge incineration, gasification and pyrolysis for a sustainable sludge-to-energy management in Greece. Waste Manag. 2014, 34, (2), 411-20.

13. Peccia, J.; Westerhoff, P., We should expect more out of our sewage sludge. Environ. Sci. Technol. 2015, 49, (14), 8271-6.

14. Ivar do Sul, J. A.; Costa, M. F., Plastic pollution risks in an estuarine conservation unit. J. Coast. Res. 2013, 65, 48-53.

15. Andrady, A. L., Microplastics in the marine environment. Mar. Pollut. Bull. 2011, 62, (8), 1596-605.

16. Zhao, S.; Zhu, L.; Wang, T.; Li, D., Suspended microplastics in the surface water of the Yangtze Estuary System, China: first observations on occurrence, distribution. Mar. Pollut. Bull. 2014, 86, (1-2), 562-568.

17. Eerkes-Medrano, D.; Thompson, R. C.; Aldridge, D. C., Microplastics in freshwater systems: A review of the emerging threats, identification of knowledge gaps and prioritisation of research needs. Water Res. 2015, 75, 63-82.

18. Dris, R.; Imhof, H.; Sanchez, W.; Gasperi, J.; Galgani, F.; Tassin, B.; Laforsch, C., Beyond the ocean: Contamination of freshwater ecosystems with (micro-) plastic particles. Environ. Chem. 2015, 12, 32. 
19. Lechner, A.; Keckeis, H.; Lumesberger-Loisl, F.; Zens, B.; Krusch, R.; Tritthart, M.; Glas, M.; Schludermann, E., The Danube so colourful: a potpourri of plastic litter outnumbers fish larvae in Europe's second largest river. Environ. Pollut. 2014, 188, 177-81.

20. McCormick, A.; Hoellein, T. J.; Mason, S. A.; Schluep, J.; Kelly, J. J., Microplastic is an abundant and distinct microbial habitat in an urban river. Environ. Sci. Technol. 2014, 48, (20), 11863-71.

21. Zhang, K.; Gong, W.; Lv, J.; Xiong, X.; Wu, C., Accumulation of floating microplastics behind the Three Gorges Dam. Environ. Pollut. 2015, 204, 117-23.

22. Talvitie, J.; Mikola, A.; Setala, O.; Heinonen, M.; Koistinen, A., How well is microlitter purified from wastewater? - A detailed study on the stepwise removal of microlitter in a tertiary level wastewater treatment plant. Water Res. 2017, 109, 164-172.

23. Hidalgo-Ruz, V.; Gutow, L.; Thompson, R. C.; Thiel, M., Microplastics in the marine environment: a review of the methods used for identification and quantification. Environ. Sci. Technol. 2012, 46, (6), 3060-75.

24. Imhof, H. K.; Ivleva, N. P.; Schmid, J.; Niessner, R.; Laforsch, C., Contamination of beach sediments of a subalpine lake with microplastic particles. Curr. Biol. 2013, 23, (19), R8678.

25. Browne, M. A.; Crump, P.; Niven, S. J.; Teuten, E.; Tonkin, A.; Galloway, T.; Thompson, R., Accumulation of microplastic on shorelines woldwide: sources and sinks. Environ. Sci. Technol. 2011, 45, (21), 9175-9.

26. Cole, M.; Lindeque, P.; Halsband, C.; Galloway, T. S., Microplastics as contaminants in the marine environment: a review. Mar. Pollut. Bull. 2011, 62, (12), 2588-97.

27. Hinojosa, I. A.; Thiel, M., Floating marine debris in fjords, gulfs and channels of southern Chile. Mar. Pollut. Bull. 2009, 58, (3), 341-50.

28. Mato, Y.; Isobe, T.; Takada, H.; Kanehiro, H.; Ohtake, C.; Kaminuma, T., Plastic resin pellets as a transport medium for toxic chemicals in the marine environment. Environ. Sci. Technol. 2001, 35, (2), 318-24.

29. Wu, B.; Taylor, C. M.; Knappe, D. R.; Nanny, M. A.; Barlaz, M. A., Factors controlling alkylbenzene sorption to municipal solid waste. Environ. Sci. Technol. 2001, 35, (22), 4569-76.

30. Müller, J. F.; Manomanii, K.; Mortimer, M. R.; McLachlan, M. S., Partitioning of polycyclic aromatic hydrocarbons in the polyethylene/water system. Fresenius J. Anal. Chem. 2001, 371, (6), 816-822.

31. Mato, Y.; Takada, H.; P., Z. M.; Kanehiro, H.; Y., K., Toxic chemicals contained in plastic resin pellets in the marine environment: Spatial difference in pollutant concentrations and the effects of resin type. Kankyo Kagakukaishi 2002, (15), 415-423.

32. $\quad$ Endo, S.; Takizawa, R.; Okuda, K.; Takada, H.; Chiba, K.; Kanehiro, H.; Ogi, H.; Yamashita, R.; Date, T., Concentration of polychlorinated biphenyls (PCBs) in beached resin pellets: Variability among individual particles and regional differences. Mar. Pollut. Bull. 2005, 50, (10), 1103-1114.

33. Pascall, M. A.; Zabik, M. E.; Zabik, M. J.; Hernandez, R. J., Uptake of polychlorinated biphenyls (PCBs) from an aqueous medium by polyethylene, polyvinyl chloride, and polystyrene films. J. Agric. Food Chem. 2005, 53, (1), 164-9.

34. Teuten, E. L.; Rowland, S. J.; Galloway, T. S.; Thompson, R. C., Potential for plastics to transport hydrophobic contaminants. Environ. Sci. Technol. 2007, 41, (22), 7759-64. 
35. Karapanagioti, H. K.; Klontza, I., Testing phenanthrene distribution properties of virgin plastic pellets and plastic eroded pellets found on Lesvos island beaches (Greece). Mar. Environ. Res. 2008, 65, (4), 283-290.

36. Saquing, J. M.; Saquing, C. D.; Knappe, D. R.; Barlaz, M. A., Impact of plastics on fate and transport of organic contaminants in landfills. Environ. Sci. Technol. 2010, 44, (16), 6396402.

37. Rochman, C. M.; Hoh, E.; Hentschel, B. T.; Kaye, S., Long-term field measurement of sorption of organic contaminants to five types of plastic pellets: implications for plastic marine debris. Environ. Sci. Technol. 2013, 47, (3), 1646-54.

38. Holmes, L. A.; Turner, A.; Thompson, R. C., Adsorption of trace metals to plastic resin pellets in the marine environment. Environ. Pollut. (Barking, Essex : 1987) 2012, 160, (1), 42-8.

39. Fries, E.; Zarfl, C., Sorption of polycyclic aromatic hydrocarbons (PAHs) to low and high density polyethylene (PE). Environ. Sci. Pollut. Res. 2012, 19, (4), 1296-1304.

40. Rochman, C. M.; Manzano, C.; Hentschel, B. T.; Simonich, S. L.; Hoh, E., Polystyrene plastic: a source and sink for polycyclic aromatic hydrocarbons in the marine environment. Environ. Sci. Technol. 2013, 47, (24), 13976-84.

41. Bakir, A.; Rowland, S. J.; Thompson, R. C., Transport of persistent organic pollutants by microplastics in estuarine conditions. Estuar. Coast Shelf Sci. 2014, 140, 14-21.

42. Rochman, C. M.; Hentschel, B. T.; Teh, S. J., Long-term sorption of metals is similar among plastic types: implications for plastic debris in aquatic environments. PLoS One 2014, 9, (1).

43. Lee, H.; Shim, W. J.; Kwon, J. H., Sorption capacity of plastic debris for hydrophobic organic chemicals. Sci. Total Environ. 2014, 470-471, 470-471.

44. Velzeboer, I.; Kwadijk, C. J. A. F.; Koelmans, A. A., Strong sorption of PCBs to nanoplastics, microplastics, carbon nanotubes, and fullerenes. Environ. Sci. Technol. 2014, 48, (9), 4869-4876.

45. Napper, I. E.; Bakir, A.; Rowland, S. J.; Thompson, R. C., Characterisation, quantity and sorptive properties of microplastics extracted from cosmetics. Mar. Pollut. Bull. 2015, 99, (1-2), 178-185.

46. Wang, F.; Shih, K. M.; Li, X. Y., The partition behavior of perfluorooctanesulfonate (PFOS) and perfluorooctanesulfonamide (FOSA) on microplastics. Chemosphere. 2015, 119, 841-847.

47. Crawford, C. B.; Quinn, B., Microplastic pollutants. 2017.

48. Liu, L.; Fokkink, R.; Koelmans, A. A., Sorption of polycyclic aromatic hydrocarbons to polystyrene nanoplastic. Environ. Toxicol. Chem. 2016, 35, (7), 1650-1655.

49. Hüffer, T.; Hofmann, T., Sorption of non-polar organic compounds by micro-sized plastic particles in aqueous solution. Environ. Pollut. 2016, 214, (17), 194-201.

50. Wu, C.; Zhang, K.; Huang, X.; Liu, J., Sorption of pharmaceuticals and personal care products to polyethylene debris. Environ. Sci. Pollut. Res. 2016, 23, (9), 8819-26.

51. Zhan, Z.; Wang, J.; Peng, J.; Xie, Q.; Huang, Y.; Gao, Y., Sorption of 3,3,4,4tetrachlorobiphenyl by microplastics: A case study of polypropylene. Mar. Pollut. Bull. 2016, 110, (1), 559-563.

52. Brennecke, D.; Duarte, B.; Paiva, F.; Caçador, I.; Canning-Clode, J., Microplastics as vector for heavy metal contamination from the marine environment. Estuar. Coast Shelf Sci. 2016, 178, 189-195. 
53. Hu, J.-Q.; Yang, S.-Z.; Guo, L.; Xu, X.; Yao, T.; Xie, F., Microscopic investigation on the adsorption of lubrication oil on microplastics. J. Mol. Liq. 2017, 227, 351-355.

54. Wang, W.; Wang, J., Different partition of polycyclic aromatic hydrocarbon on environmental particulates in freshwater: Microplastics in comparison to natural sediment. Ecotoxicol. Environ. Saf. 2017, 147, (Supplement C), 648-655. 